Methodology article

Open Access

\title{
Comparison of two chromogenic media and evaluation of two molecular based identification systems for Enterobacter sakazakii detection
}

\author{
Angelika Lehner ${ }^{1}$, Sabine Nitzsche ${ }^{1}$, Pieter Breeuwer ${ }^{2}$, Benjamin Diep², \\ Karin Thelen ${ }^{3}$ and Roger Stephan*1
}

Address: ${ }^{1}$ Institute for Food Safety and Hygiene, Vetsuisse Faculty, University of Zurich, CH-8057 Zurich, Switzerland, ${ }^{2}$ Nestle Research Center, Nestec Ltd, Ver-Ches-les-Blanc, Lausanne, Switzerland and 3vermicon AG, 80992, Munich, Germany

Email: Angelika Lehner - lehnera@fsafety.unizh.ch; Sabine Nitzsche - sabinenitzsche@ hotmail.com;

Pieter Breeuwer - pieter.breeuwer@rdls.nestle.com; Benjamin Diep - Benjamin.Diep@rdls.nestle.com; Karin Thelen - thelen@vermicon.com; Roger Stephan* - stephanr@fsafety.unizh.ch

* Corresponding author

Published: 23 February 2006

BMC Microbiology 2006, 6:15 doi:10.1/86/147|-2180-6-15
Received: 16 December 2005

Accepted: 23 February 2006

This article is available from: http://www.biomedcentral.com/I47I-2/80/6/15

(C) 2006 Lehner et al; licensee BioMed Central Ltd.

This is an Open Access article distributed under the terms of the Creative Commons Attribution License (http://creativecommons.org/licenses/by/2.0), which permits unrestricted use, distribution, and reproduction in any medium, provided the original work is properly cited.

\begin{abstract}
Background: Enterobacter sakazakii is a foodborne pathogen that has been associated with sporadic cases and outbreaks causing meningitis, necrotizing enterocolitis and sepsis especially in neonates. The current FDA detection method includes two enrichment steps, the subculturing of the second enrichment broth on a selective agar (VRBG), a further subculturing of selected grown colonies on TSA and the subsequent biochemical identification of yellow-pigmented colonies by API20E. However, there is a strong need for simplified methods for isolation and identification of E. sakazakii. In this study, two chromogenic media, which allow to indicate presumptive $E$. sakazakii colonies by the alpha glucosidase activity, as well as a newly developed I,6-alpha-glucosidase based conventional PCR assay and a rRNA oligonucleotide probe based commercial test system for identification of presumptive $E$. sakazakii were evaluated on 98 target and non-target strains. The methods were compared with respect to specificity aspects.
\end{abstract}

Results: A total of 75 presumptive E. sakazakii and 23 non-target strains were analysed by using chromogenic media, alpha-glucosidase based PCR assay, and the VIT assay. For most presumptive E. sakazakii strains on the chromogenic media, the PCR and VIT assay confirmed the identification. However, for a number of presumptive $E$. sakazakii isolates from fruit powder, the alphaglucosidase PCR and VIT assay did not correspond to the typical E. sakazakii colonies on DFI and ESIA. Further characterization by API32E identification, phylogenetic analysis of partial $16 \mathrm{~S}$ rRNA sequences and ribotyping strongly suggested, that these strains did not belong to the species $E$. sakazakii. The newly developed alpha-glucosidase based PCR assay as well as the commercially available VIT Enterobacter sakazakii identification test showed an excellent correlation with the I6S rRNA data, and are thus well suited for identification of $E$. sakazakii.

Conclusion: The results indicate that presumptive colonies on ESIA and DFI media need further species identification. Both evaluated molecular methods, the alpha-glucosidase PCR and the I6S RNA in situ hybridisation test (VIT), although based on completely different target regions and methodologies performed equally well in terms of specificity. 


\section{Background}

E. sakazakii is a motile, non-spore forming, Gram negative facultative anaerobe microorganism. It was previously referred to as "yellow-pigmented Enterobacter cloacae" until it was designated a unique species by Farmer et al. [1].

The organism is an occasional contaminant of powdered infant formula milk that can cause a rare, but life threatening form of neonatal meningitis and necrotizing enterocolitis, which is the most common gastrointestinal emergency in the newborn $[2,3]$.

The current Food and Drug Administration (FDA) method for detection of E. sakazakii includes a pre-enrichment procedure in buffered peptone water (BPW), enrichment in Enterobacteriaceae enrichment (EE) broth, plating on violet red bile glucose agar (VRBG) and picking of five grown colonies onto tryptone soy agar (TSA) plates, which are incubated at $25^{\circ} \mathrm{C}$ for $48-72$ hours. Yellow-pigmented colonies, typical for E. sakazakii, on the TSA plates are confirmed using the API20E system. However, there is a strong need for simplified methods for isolation and identification of E. sakazakii. Guillaume et al. [4] published a new procedure, based on selective enrichment in modified lauryl sulfate tryptone broth, by addition of 0.5 $\mathrm{M} \mathrm{NaCl}$. Additionally, there is a strong need for good identification methods for presumptive E. sakazakii isolates. A feature, which can be used for this is the $\alpha$-glucosidase activity, which was demonstrated to be present in all E. sakazakii strains and not in most other members of the Enterobacteriaceae [5]. Based on this biochemical property several differential media were developed recently [6-9]. Two of these media the Oxoid Chromogenic Enterobacter sakazakii Agar (Oxoid CM1055, Oxoid, UK) - also known as the Druggan-Forsythe-Iversen (DFI) formulation [7] -

Table I: Target and non-target strains used in this study and results obtained with the chromogenic media DFI and ESIA, the $\alpha$ glucosidase based PCR assay and the rRNA probe based VIT test.

\begin{tabular}{|c|c|c|c|c|c|}
\hline Species (number of strains) & Origin & ESIA* & $\mathrm{DFI}^{+}$ & PCR & VIT \\
\hline E. cloacae (I) & ATCCI 3047 & neg & neg & neg & neg \\
\hline E. cloacae (I) & LMG3008 & neg & neg & neg & neg \\
\hline E. cloacae (6) & clinical, cosmetics & neg & neg & neg & neg \\
\hline E. cloacae (2) & food & neg & neg & neg & neg \\
\hline E. dissolvens (I) & ATCC23373 & neg & neg & neg & neg \\
\hline E. hermanii (I) & wild strain & neg & neg & neg & neg \\
\hline P. agglomerans (I) & ATCC27I55 & neg & neg & neg & neg \\
\hline P. agglomerans (2) & food, cosmetics & neg & neg & neg & neg \\
\hline Pantoea sp. (I) & food & neg & neg & neg & neg \\
\hline K. oxytoca (I) & cheese & neg & neg & neg & neg \\
\hline S. liquefaciens (I) & cosmetics & neg & neg & neg & neg \\
\hline S. ficaria (I) & production environment & neg & pos & neg & neg \\
\hline Serratia sp. (I) & cosmetics & neg & neg & neg & neg \\
\hline Salmonella Enterititis (I) & wild strain & neg & neg & neg & neg \\
\hline S. sonnei (I) & wild strain & neg & neg & neg & neg \\
\hline E. coli (I) & ATCC25922 & neg & neg & neg & neg \\
\hline E. sakazakii (I) & ATCC29544 & pos & pos & pos & pos \\
\hline E. sakazakii (I) & ATCC29004 & pos & pos & pos & pos \\
\hline E. sakazakii (I) & ATCC5I329 & pos & pos & pos & pos \\
\hline E. sakazakii (20) & fruit powderl & pos & pos & pos & pos \\
\hline E. sakazakii (5) & cosmetics & pos & pos & pos & pos \\
\hline E. sakazakii (I5) & human ${ }^{2}$ & pos & pos & pos & pos \\
\hline E. sakazakii (I) & milk powder 3 & pos & pos & pos & pos \\
\hline E. sakazakii (2) & baby food 4 & pos & pos & pos & pos \\
\hline E. sakazakii (I) & milk 5 & pos & pos & pos & pos \\
\hline E. sakazakii (15) & production environment ${ }^{6}$ & pos & pos & pos & pos \\
\hline E. sakazakii (I) & production environment & pos & neg & pos & pos \\
\hline
\end{tabular}

$*$ positive $=$ turquoise colonies typical for $E$. sakazakii

+ positive = blue-green coloured colonies typical for E. sakazakii

lincluding strains (Acc. number): 858 (AY752936), 759 (AY752939), 954 (AY752938), AY752943

2including strains (Acc. number): ES II (AY803।87), ES 4 (AY803।86)

3including strain (Acc. number): FSM 265 (AY803191)

${ }^{4}$ including strain (Acc. number): FSM 393 (AY75294I)

5including strain (Acc. number): FSM 33 (AY752940)

${ }^{6}$ including strains (Acc. number): FSM 468 (AY752942), FSM 266 (AY803190) 
Table 2: Colonies on ESIA and DFI media, growth in $\mathrm{mLST}$ broth and results of the two molecular identification methods for the 12 non-E.sakazakii fruit powder isolates investigated in this study.

\begin{tabular}{llllll}
\hline Fruit powder strain & colonies on ESIA* & colonies on $\mathrm{DFI}^{+}$ & $\begin{array}{l}\text { growth in } \mathrm{mLST} \text { at } \\
44^{\circ} \mathrm{C}\end{array}$ & $\begin{array}{l}\alpha \text {-glucosidase PCR } \\
\text { VIT test }\end{array}$ \\
\hline $947 / 03$ & & no growth & neg & neg \\
$1129 / 04$ & non typical & typical & no growth & neg & neg \\
$1159 / 04$ & typical & typical & no growth & neg & neg \\
$1160 / 04$ & typical & typical & no growth & neg & neg \\
$508 / 05$ & typical & typical & no growth & neg & neg \\
$509 / 05$ & non typical & typical & no growth & neg & neg \\
$513 / 05$ & typical & typical & no growth & neg & neg \\
$516 / 05$ & typical & typical & no growth & neg & neg \\
$517 / 05$ & typical & non typical & no growth & neg & neg \\
$601 / 05$ & typical & typical & no growth & neg & neg \\
$603 / 05$ & typical & typical & no growth & neg & neg \\
$610 / 05$ & non typical & typical & no growth & neg & neg
\end{tabular}

* typical $=$ turquoise colonies typical for E. sakazakii

+ typical = blue-green coloured colonies typical for E. sakazakii

and Enterobacter sakazakii Isolation Agar (ESIA, AES, France), are commercially available and were included in our comparative study.

In a recent study, the molecular basis of the $\alpha$-glucosidase activity in E. sakazakii was determined [10]. An open reading frame containing a sequence potentially coding for a 1,6 - $\alpha$-glucosidase was identified. In the current study, the potential of a PCR system based on the 1,6- $\alpha$-glucosidase for the specific identification of $E$. sakazakii was evaluated on the DNA of target and non-target strains.

The VIT (vermicon identification technology) represents a commercially available detection and identification system based on fluorescently labelled gene probes targeting specified regions on the ribosomal RNA of the bacteria. Subsequent to analysis, the illuminated E. sakazakii cells are visualised under an epifluorescence microscope.

The current study represents a comparison of two commercial chromogenic media, which indicate presumptive E. sakazakii colonies, as well as an evaluation of an $\alpha$-glucosidase based PCR assay and a rRNA targeting in situ technique for the rapid identification of E. sakazakii.

Ninety-eight target and non-target strains were included in the study and results were compared for specificity and convenience in performance.

\section{Results and discussion \\ The chromogenic media}

The results of the target and non-target strains included in this study for the comparison of the DFI and the ESIA media are summarized in table 1 and 2 in view of producing presumptive, typical colonies for E. sakazakii. Gener- ally, the results for the 63 E. sakazakii strains were in good concordance, blue-green coloured colonies on DFI agar and turquoise on ESIA media, except for one E. sakazakii strain (FSM 322), which gave grey-white colonies on DFI agar (Figure 1A) and thus was considered negative on this medium. Furthermore, of the 23 non-target strains (Table 1), one S. ficaria environmental isolate showed the typical blue-green colour when grown on DFI agar, but was correctly identified as negative on ESIA medium (Figure 1B).

However, within a set of 12 strains, originally isolated from fruit powder (Table 2), typical blue green and turquoise colonies, respectively were observed for 8 strains on ESIA and for 11 isolates on DFI medium, when incubated at the recommended temperature. An example of the fruit powder isolate 1160/04 grown on DFI and ESIA medium is given in Figure 2A and 2B. API32E analysis on these strains revealed ambiguous results, but none of the strains were identified as E. sakazakii. According to the literature P. shigelloides, E. vulneris, C. koseri or Pantoea sp. can give typical coloured colonies on DFI agar [6]. API32E identification suggested as identification $\mathrm{E}$. vulneris and Pantoea spp. as well as Buttiauxiella agrestis [11], which was previously known as Citrobacter group F, for several isolates within this group, although with low levels of confidence. However, the two reference strains E. vulneris ATCC 33821 and P. agglomerans ATCC 27155, included in this study were correctly identified as negative by both selective media (Table 1).

In order to obtain more information on the fruit powder strains partial sequencing of the 16S rRNA gene was performed. Affiliation to the phylogenetic tree revealed, that all isolates were clearly distinct from E. sakazakii sequences from both lineages [12]. In Figure 3 and 4 the 
$1 \mathrm{~A}$

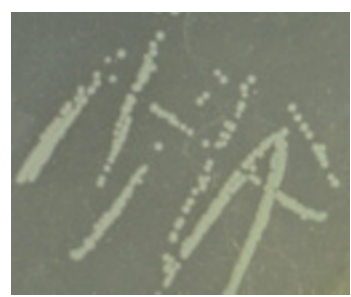

Figure I

Atypical colonies of E. sakazakii FSM 322 (A) and typical colonies of S. ficaria (B) on DFI agar.

phylogenetic tree (Figure 3) and the respective distances matrix (Figure 4) of these strains with respect to E. sakazakii strains from both lineages and other selected members of the Enterobacteriaceae are given. Sequence similarities of $96-97 \%$ to type strains of other members of the Enterobacteriacaeae suggested that the organisms were members of the same genus but none of the isolates exhibited sequence similarities $>97 \%$ to E. sakazakii sequences from both lineages (Figure 4). Finally, ribotyping was performed on nine of the questionable strains again revealing, that the strains are not belonging to the E. sakazakii group (data not shown).

Additionally, growth experiments were performed for the strains of interest using a recently developed selective enrichment for E. sakazakii detection in environmental samples [4]. Experiments were performed in lauryl sulfate tryptose broth (mLST) supplemented with $0.5 \mathrm{M} \mathrm{NaCl}$ and $10 \mathrm{mg} /$ liter vacomycin with or without an included non-selective enrichment step in BPW starting with BHI grown cultures. All strains were unable to grow within mLST broth at $44^{\circ} \mathrm{C}$, regardless including a non-selective enrichment step in BPW or not (Table 2). In the study recently published by Guillaume-Gentil et al. [4], all of the E. sakazakii strains $(\mathrm{n}=99)$ tested, were able to grow in mLST at $45^{\circ} \mathrm{C}$, whereas 35 of 39 strains of potential competitors, all belonging to the Enterobacteriaceae, were suppressed. They concluded, that the inclusion of this enrichment broth could be very useful for the reliable detection of E. sakazakii in environmental samples. In our study, however, E. sakazakii strain FSM 322 could also not grow in mLST.

\section{The $\alpha$-glucosidase based PCR}

Applying the $\alpha$-glucosidase based PCR system we were able to identify all target strains. No false positive result was obtained within the group of non-target strains. An example of the identification of a fruit powder strain by PCR assay is given in Figure 5A.
$2 \mathrm{~A}$

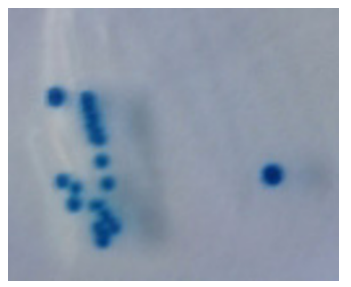

2B

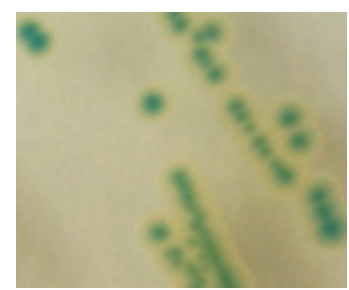

Figure 2

Non-E. sakazakii fruit powder isolate I I60/04 grown as typical colonies on ESIA (A) and DFI (B) media.

It is worth mentioning that both compared selective media are based on the proposed $\alpha$-glucosidase activity of E. sakazakii. From literature it is known, that several other organisms also exhibit this biochemical feature and can grown on DFI [7]. The $\alpha$-glucosidase based PCR system, however, exclusively targets the gene responsible for the $\alpha$-glucosidase activity in E. sakazakii.

\section{VIT (vermicon identification technology)}

By using the VIT test, all of the E. sakazakii strains were identified. No false positive result was obtained within the group of non-target strains. These results were in good concordance with those obtained with the $\alpha$-glucosidase based PCR system. The target strains were easy to identify due to the specific red colour under the epifluorescence microscope. In Figure 5B, an example of a positive identification of E. sakazakii fruit powder strain $1160 / 04$ by VIT is given. However, during analysis of the strains used in the study, some autofluorescence was observed in several samples of the non-target strains. Inclusion of a positive reference strain during analysis can overcome this problem, since a "true signal" differs significantly in brightness.

The major advantage of this easy to handle molecular method is represented by the fact, that the bacterial rRNA is targeted by the probes, thus in principle only vital $E$. sakazakii cells containing an efficiently high ribosomal content are detected by the assay. Results are obtained within three hours and the performance of the test is not restricted to an especially equipped laboratory.

Detection is possible to the single cell level, even in mixed material containing target and non-target cells. The detection limit of the assay was experimentally determined using serial dilutions of overnight cultures of two different E. sakazakii reference strains (ATCC29544, ATCC51329) and determination of the $\mathrm{cfu} / \mathrm{ml}$ by quantitative plating. 


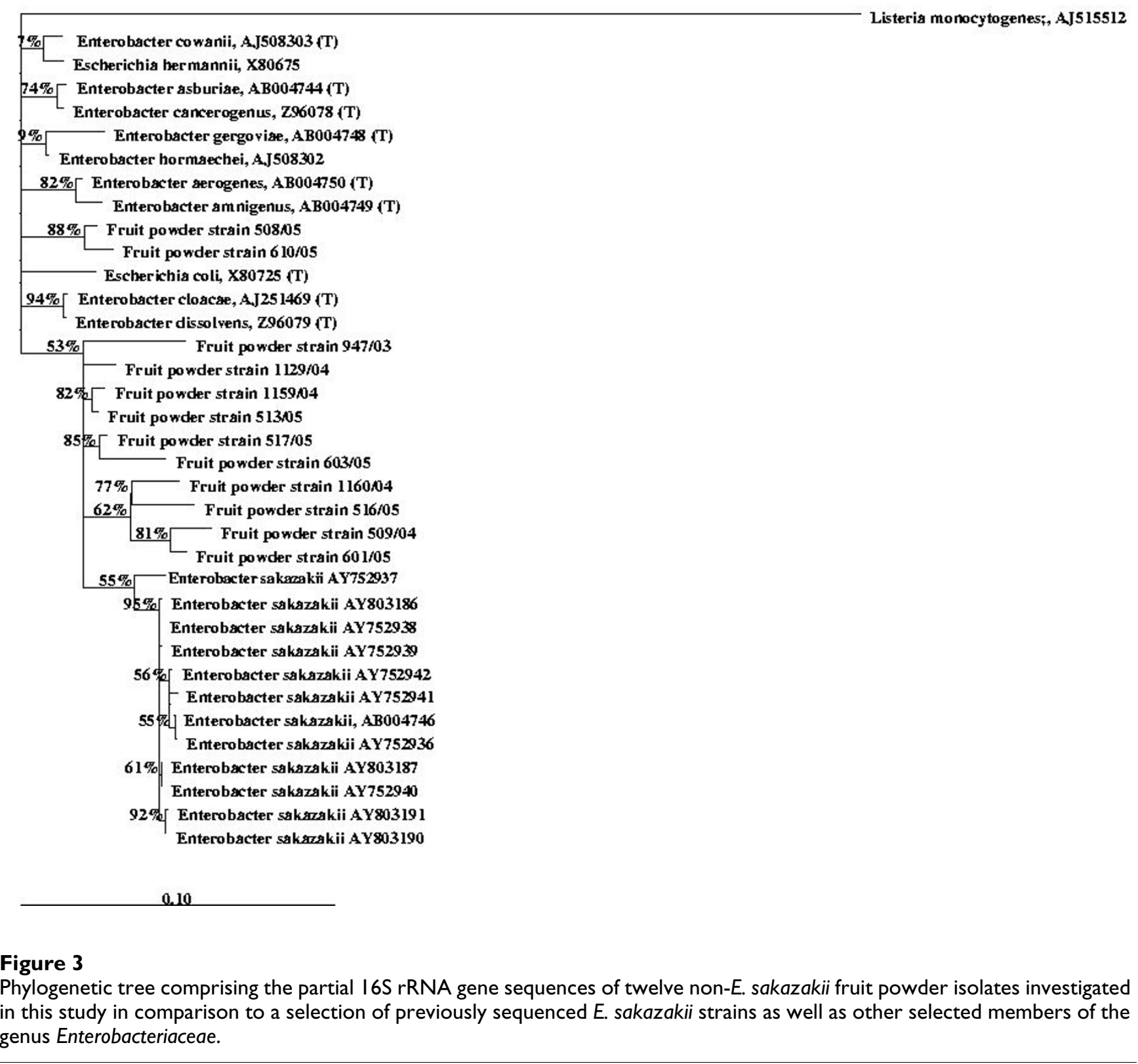

A detection limit of $10^{3} \mathrm{cfu} / \mathrm{ml}$ was ascertained for both strains.

\section{Conclusion}

By comparing the two chromogenic media on a set of 98 target and non-target strains, the ESIA medium proved to be more indicative for presumptive E. sakazakii colonies. Inclusion of an enrichment step in mLST can reduce the number of presumptive colonies, with are not E. sakazakii. Nevertheless, all presumptive colonies on ESIA and DFI media need a further species identification. The two molecular methods, although based on completely different target regions and methodologies performed equally well in identifying E. sakazakii strains. Both methods showed $100 \%$ specificity. The PCR based system needs a DNA extraction step, prior to amplification set up, but is easily implemented into PCR adapted laboratories. The VIT test represented a fast and convenient to handle test system.

\section{Methods \\ Target strains}

63 E. sakazakii strains from human, food, cosmetics and environmental origin including three reference strains were used in the study (Table 1). 


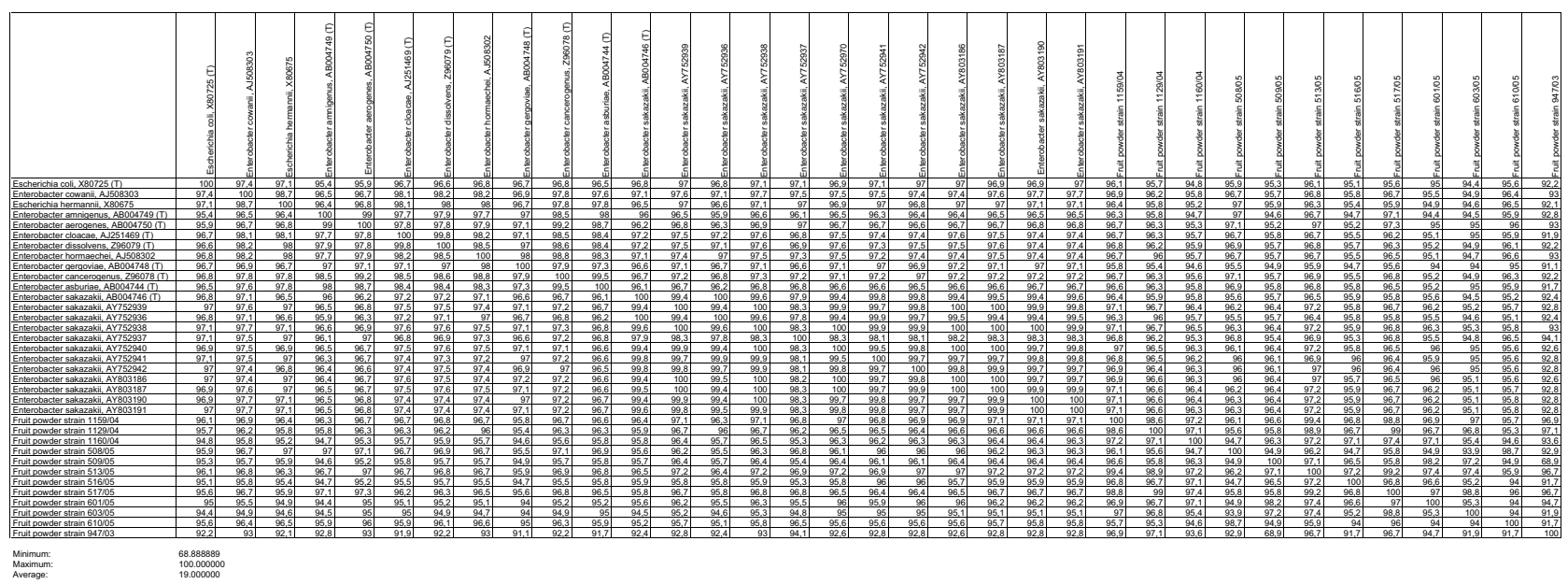

Figure 4

Respective distances matrix calculated with the sequences from figure 3.

\section{Non-target strains}

A first set of 39 strains belonging to different species other than E. sakazakii represented a part of the non-target group (Table 1).

Moreover, another 12 strains were added to this non-target group (table 2). They were isolated from fruit powder using the FDA approach. All of these strains have shown typical colonies on ESIA and/or DFI agar and were shown by API32E to be non-E. sakazakii. However, it was not possible to reliable identify theses strains by API32E. In order to do this, 16S rRNA gene sequencing and phylogenetic analysis of these fruit powder strains was done. For PCR amplification of the 16S rRNA gene, DNA was extracted from $1 \mathrm{ml}$ cultures grown in BHI broth for 24 hours at $37^{\circ} \mathrm{C}$ using the DNeasy Tissue Kit (Qiagen AG, Switzerland) in accordance with the protocol of the supplier. Partial sequencing of the 16S rRNA genes of these strains was performed according to the method recently published by Lehner et al. [12]. The same primers were used for amplification and sequencing of the 16S rRNA gene, except that no internal walking primers were employed. The partial $16 \mathrm{~S}$ rRNA gene sequences of the 12 strains were added to an alignment of 28.000 almost full length small subunit rRNA sequences by using the alignment tool of the ARB program package. Phylogenetic analyses were performed using distance matrix and the TREEPUZZLE tool included

\section{$5 \mathrm{~A}$}

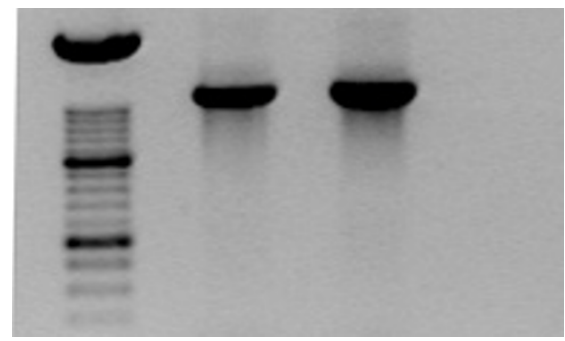

$5 B$

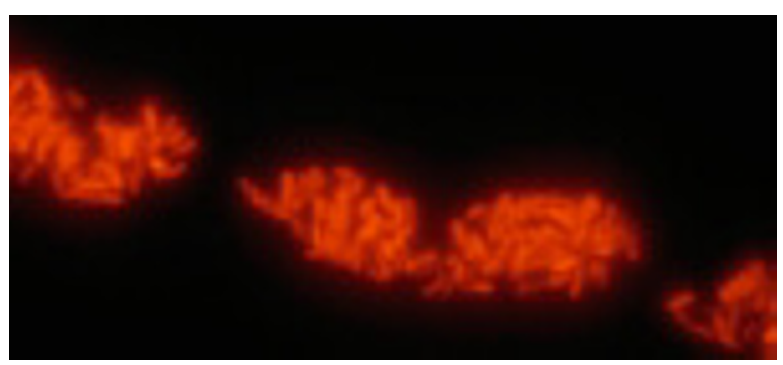

\section{Figure 5}

(A): Strain II60/04 identified as E. sakazakii by the $\alpha$-glucosidase based PCR assay (A); lane I: DNA 100 bp marker, lane 2: positive control (E. sakazakii reference strain ATCC 29004), lane 3: E. sakazakii fruit powder isolate FSM 322, lane 4: negative control. (B) Positive VIT result of strain FSM 322 
in the ARB software package employing special data structures (PT-servers) derived from the ssu-rRNA database „ssu_jan04.arb.

Additionally, ribotyping was performed on nine selected fruit powder strains $(1159 / 04,947 / 03,1129 / 04,517 / 05$, $610 / 05,1160 / 04,513 / 05,509 / 05,508 / 05)$ on a DuPont RiboPrinter Microbial Characterization System ${ }^{T M}$ according to the method described by Bruce [13].

Furthermore, growth of the fruit powder strains in lauryl sulfate tryprose broth supplemented with $0.5 \mathrm{M} \mathrm{NaCl}$ and $10 \mathrm{mg} /$ liter vancomycin (mLST) for $22-24 \mathrm{~h}$ at $44 \pm$ $0.5^{\circ} \mathrm{C} \mathrm{[4]} \mathrm{was} \mathrm{examined} \mathrm{in} \mathrm{two} \mathrm{different} \mathrm{manners:} \mathrm{i)} \mathrm{inoc-}$ ulating $10 \mathrm{ml}$ of mLST broth with $10-100 \mathrm{cfu}$ of a BHI grown culture (overnight, $37^{\circ} \mathrm{C}$ ), ii) inoculating $25 \mathrm{ml}$ of buffered peptone water (BPW) $\left(37^{\circ} \mathrm{C}\right.$ for $\left.16-20 \mathrm{~h}\right)$ with $10-100 \mathrm{cfu}$ of a BHI grown culture (overnight, $37^{\circ} \mathrm{C}$ ). Thereafter $10 \mathrm{ml}$ of the mLST broth were inoculated with $0.1 \mathrm{ml}$ of the BPW grown culture and incubated as described above.

\section{Chromogenic media}

The strains were streaked onto DFI agar plates (Oxoid, UK) and ESIA agar plates (AES, France) and incubated at $37^{\circ} \mathrm{C}$ (DFI) and $44^{\circ} \mathrm{C}$ (ESIA) for $24 \mathrm{~h}$. Colonies that were entirely blue-green on DFI and turquoise on ESIA media after $24 \mathrm{~h}$ incubation were indicative for presumptive $E$. sakazakii colonies. According to the suppliers protocol purple colonies on ESIA media and non-blue green colonies on DFI agar were considered non typical.

\section{Alpha glucosidase specific PCR}

Within a recent study two open reading frames were identified both coding for enzymes with the potential to hydrolyze the fluorescent substrate 4-methylumbellyferryl- $\alpha$-D-glucoside [10]. The primers that were used for subcloning in that study were modified in the current study and the optimal annealing temperature was determined. The following primers were applied for specific amplification of the gene putatively encoding the $\alpha$-glucosidase activity: EsAgf: 5'- TGA AAG CAA TCG ACA AGA AG-3'and EsAgr: 5'- ACT CAT TAC CCC TCC TGA TG-3' generating a product of $1680 \mathrm{bp}$ in size (GenBank accession number AM075208). The PCR reactions were set up in a total volume of $50 \mu \mathrm{l}$. The reaction mixture contained $5 \mathrm{pmol}$ primers each, $100 \mu \mathrm{M}$ dNTPs each, $1 \times$ Taq polymerase buffer and 2 U Taq polymerase (Promega). Thermal cycling was carried out by using an initial denaturation step at $94^{\circ} \mathrm{C}$ for $2 \mathrm{~min}$, followed by 29 cycles of denaturation at $94^{\circ} \mathrm{C}$ for $30 \mathrm{sec}$, annealing at $58{ }^{\circ} \mathrm{C}$ for 60 sec and extension at $72^{\circ} \mathrm{C}$ for $90 \mathrm{sec}$. Cycling was completed by a final elongation step at $72^{\circ} \mathrm{C}$ for $5 \mathrm{~min}$. The amplification products were analysed by gel electrophoresis and ethidium bromide staining.

\section{Vermicon identification technology (VIT)}

The VIT Enterobacter sakazakii test (vermicon, Munich, Germany) was performed according to the instructions of the manufacturer. For analysis, $1 \mathrm{ml}$ of $\mathrm{BHI}$ grown cultures (overnight, $37^{\circ} \mathrm{C}$ ) were used.

Accession numbers of 16S rRNA genes of fruit powder strains included in the study:

Strain 610/05 (GenBank: DQ273680), strain 508/05 (GenBank: DQ273681), strain 516/05 (GenBank: DQ273682), strain 1159/04 (GenBank: DQ273683), strain 601/05 (GenBank: DQ273684), strain 603/05 (GenBank: DQ273685), strain 1160/04 (GenBank: DQ273686), strain 1129/04 (GenBank: DQ273687), strain 513/05 (GenBank: DQ273688), strain 517/05 (GenBank: 273689), strain 509/05 (GenBank: DQ273690), strain 947/03 (GenBank: DQ273691)

\section{Authors' contributions}

AL carried out the PCR experiments, the phylogenetic analyses and drafted the manuscript. SN participated in the PCR experiments and carried out the cultural and VIT experiments. PB and BD performed ribotyping and participated in the cultural experiments. KT carried out the $16 \mathrm{~S}$ rRNA gene sequencing. RS conceived of the study, participated in its design and coordination and helped to draft the manuscript. All authors read and approved the final manuscript.

\section{References}

I. Farmer JJ, Ashbury MA, Hickman FW, Brenner DJ, The Enterobacteriaceae Group: Enterobacter sakazakii : a new species of Enterobacteriaceae isolated from clinical species. Int J Syst Bacteriol 1980, 30:569-584.

2. Bar-Oz B, Preminger A, Peleg O, Block C, Arad I: Enterobacter sakazakii infection in the newborn. Acta Paediatr 200I, 90:356-358.

3. van Acker J, de Smet F, Muyldermans G, Bougatef A, Naesens A, Lauwers S: Outbreak of necotizing enterocolitis associated with Enterobacter sakazakii in powdered milk formula. J Clin Microbiol 200I, 39:293-297.

4. Guillaume-Gentil O, Sonnard V, Kandhai MC, Marugg JD, Joosten H: A simple and rapid culture method for detection of Enterobacter sakazakii in environmental samples. J Food Prot 2005, 68:64-69.

5. Muytjens HL, van der Ros-van der Repe J, van Druten HA: Enzymatic profiles of Enterobacter sakazakii and related species with special reference to the alpha-glucosidase reaction and reproducibility of the test system. J Clin Microbiol 1984, 20:684-686

6. Leuschner RG, Baird F, Donald B, Cox LJ: A medium for the presumptive detection of Enterobacter sakazakii in infant formula. Food Microbiol 2004, $21: 527-533$.

7. Iversen $\mathrm{C}$, Druggan $\mathrm{P}$, Forsythe $\mathrm{S}$ : A selective medium for Enterobacter sakazakii, a preliminary study. Int J Food Microbiol 2004, 96:133-139.

8. Oh SE, Kang $\mathrm{DH}$ : Fluorogenic selective and differential medium for isolation of Enterobacter sakazakii . Appl Environ Microbiol 2004, 70:5692-5694.

9. Kandhai MC, Reij MW, Gorris LG, Guillaume-Gentil O, Beumer RR, van Shothorst M: A new protocol for the detection of Enterobacter sakazakii applied to environmental samples. J Food Prot 2004, 67: 1267-1270. 
10. Lehner A, Riedel K, Rattei T, Ruepp A, Frishman D, Breeuwer P, Diep $B$, Eberl L, Stephan R: Molecular characterization of the alpha glucosidase activity in Enterobacter sakazakii reveals the presence of a putative gene cluster for palatinose metabolism. Syst Appl Microbiol in press.

II. Ferragut C, Izard D, Gavuni F, Lefebrve B, Leclerc H: Buttiauxella, a new genus of the family Enterobacteriaceae. Int J Syst Bacteriol 1982, 32:266-268.

12. Lehner A, Tasara T, Stephan R: I6S rRNA gene based analysis of Enterobacter sakazakii strains from different sources and development of a PCR assay for identification. BMC Microbiol 2004, 4:43.

13. Bruce J: Automated system rapidly identifies and characterizes microorganisms in food. Food Technol 1996, 50:77-78.

Publish with Bio Med Central and every scientist can read your work free of charge

"BioMed Central will be the most significant development for disseminating the results of biomedical research in our lifetime. "

Sir Paul Nurse, Cancer Research UK

Your research papers will be:

- available free of charge to the entire biomedical community

- peer reviewed and published immediately upon acceptance

- cited in PubMed and archived on PubMed Central

- yours - you keep the copyright

Submit your manuscript here:

http://www.biomedcentral.com/info/publishing_adv.asp 